\title{
Independent comparison study of six different electronic tongues applied for pharmaceutical analysis
}

\author{
Miriam Pein ${ }^{\mathrm{a}, *}$, Dmitry Kirsanov ${ }^{\mathrm{b}, \mathrm{c}}$, Patrycja Ciosek ${ }^{\mathrm{d}}$, Manel del Valle ${ }^{\mathrm{e}}$, \\ Irina Yaroshenko ${ }^{\mathrm{b}, \mathrm{c}}$, Małgorzata Wesoły ${ }^{\mathrm{d}}$, Marcin Zabadaj ${ }^{\mathrm{d}}$, Andreu Gonzalez-Calabuig ${ }^{\mathrm{e}}$, \\ Wojciech Wróblewski ${ }^{\mathrm{d}}$, Andrey Legin ${ }^{\mathrm{b}, \mathrm{c}}$
}

\footnotetext{
a Institute of Pharmaceutics and Biopharmaceutics, Heinrich-Heine University Duesseldorf, Universitaetsstr. 1, 40225 Duesseldorf, Germany

${ }^{b}$ Institute of Chemistry, St. Petersburg State University, Universitetskaya nab. 7/9, 199034 St. Petersburg, Russia

${ }^{\mathrm{c}}$ Laboratory of Artificial Sensory Systems, ITMO University, Kronverskiy pr. 49, 197101 St. Petersburg, Russia

${ }^{\mathrm{d}}$ Department of Microbioanalytics, Warsaw University of Technology, Noakowskiego 3, 00-664 Warsaw, Poland

e Sensors and Biosensors Group, Department of Chemistry, Universitat Autònoma de Barcelona, Edifici Cn, 08193 Bellaterra, Barcelona, Spain
}

\section{A R T I C L E I N F O}

\section{Article history:}

Received 30 January 2015

Received in revised form 15 May 2015

Accepted 28 May 2015

Available online 3 June 2015

\section{Keywords:}

Electronic tongue

Comparison

Blind conditions

Taste masking

\begin{abstract}
A B S T R A C T
Electronic tongue technology based on arrays of cross-sensitive chemical sensors and chemometric data processing has attracted a lot of researchers' attention through the last years. Several so far reported applications dealing with pharmaceutical related tasks employed different e-tongue systems to address different objectives. In this situation, it is hard to judge on the benefits and drawbacks of particular e-tongue implementations for R\&D in pharmaceutics. The objective of this study was to compare the performance of six different e-tongues applied to the same set of pharmaceutical samples. For this purpose, two commercially available systems (from Insent and AlphaMOS) and four laboratory prototype systems (two potentiometric systems from Warsaw operating in flow and static modes, one potentiometric system from St. Petersburg, one voltammetric system from Barcelona) were employed. The sample set addressed in the study comprised nine different formulations based on caffeine citrate, lactose monohydrate, maltodextrine, saccharin sodium and citric acid in various combinations. To provide for the fair and unbiased comparison, samples were evaluated under blind conditions and data processing from all the systems was performed in a uniform way. Different mathematical methods were applied to judge on similarity of the e-tongues response from the samples. These were principal component analysis (PCA), $\mathrm{RV}^{\prime}$ matrix correlation coefficients and Tuckerís congruency coefficients.
\end{abstract}

(C) 2015 Elsevier B.V. All rights reserved.

\section{Introduction}

Evaluation of taste parameters and taste-masking efficacy gains importance, especially in the development of pediatric drug formulations. The traditional way to assess the taste is obviously based on human sensory panels. However, besides ethical aspects, this method is associated with numerous problems, such as sensory panel fatigue or dependency of sensory scores on the health con-

\footnotetext{
* Corresponding author. Tel.: +49 211 8114225; fax: +49 2118114251.

E-mail addresses: miriam.pein@hhu.de (M. Pein),d.kirsanov@gmail.com (D. Kirsanov), pciosek@ch.pw.edu.pl (P. Ciosek), manel.delvalle@uab.es (M. del Valle), irina.s.yaroshenko@gmail.com (I. Yaroshenko), wesolymalgorzata@gmail.com (M. Wesoły),zabadajmarcin@gmail.com (M. Zabadaj), andreu.gonzalez@uab.cat (A. Gonzalez-Calabuig), wuwu@ch.pw.edu.pl (W. Wróblewski), andrey.legin@gmail.com (A. Legin).
}

ditions of a panelist. Artificial taste assessment of pharmaceutics seems to be a very attractive alternative, since it provides a fast and objective formulation evaluation. The first works dealing with bitter taste sensing by e-tongue were presented by Toko [1]. Since then, multichannel taste sensor that later became a fundamental part of commercial taste sensing system by Insent had been used for quantification of basic taste sensations in large variety of samples [2-4]. Various studies were devoted to the application of different versions of e-tongues for the assessment of taste in pharmaceutical samples [5-16]. A variety of sensors and sensor systems were developed and applied in these research efforts, based on both, commercial $[5,6,9,10,13-15]$ or laboratory instrumentation $[7,8,11,16]$. Most of these reports are focused on the development of new drug formulations by choosing appropriate taste masking strategies. Besides, original and generic products were compared $[12,13]$, products modified with commercially available beverages or jellies characterized [14,15], the effect of micro encapsula- 
tion [16] or oral film formulations [17] on taste-masking efficacy assessed and stability and dose uniformity studies [18] presented. In a recent study, the capability of HPLC and e-tongue analysis was compared with human taste panels regarding taste assessment and the applied e-tongue was proven to be even more sensitive than the human taste panels [19].

The idea of "e-tongues" is based on the application of an array of cross-sensitive chemical sensors combined with multivariate data processing techniques to yield quantitative and qualitative information about studied sample [20]. Chemical sensors in an array can be based on various principles of signal transduction: potentiometry, voltammetry, optical sensing, surface acoustic waves, etc. The first two mentioned transduction schemes gained widest acceptance in research these days [21]. This is probably due to the ease of instrumentation required for implementation of these techniques and broad options to modify sensor response in order to fit it to particular analytical task.

The electronic tongue TS-5000Z and SA402B (Insent Inc., AtsugiShi, Japan) and those multisensor systems from the laboratories of Warsaw (Department of Microbioanalytics, Warsaw University of Technology; Poland) and St. Petersburg (Institute of Chemistry, St. Petersburg State University/Laboratory of Artificial Sensory Systems, ITMO University; Russia) are based on direct potentiometric measurements with sensors based on PVC-plasticized membranes $[4,7,8,10,16]$. The differences between these systems are in the number and composition of sensor membranes leading to the fact that all these systems have somewhat different sensitivity to the components in the analyzed media. In case of FIA version from Warsaw the kinetics of interaction of various components with membrane material is also taken into account thus providing for additional source of chemical information. The $\alpha$-Astree e-tongue (AlphaMOS, Toulouse, France) is based on ISFETs (ion-selective field effect transistors) with polymeric sensor membranes [10], which are generally similar to those employed in systems from Warsaw and St. Petersburg. However, the measuring principle is not direct potentiometric, but based on recording the feedback gate potential constantly keeping the FET current. The voltammetric e-tongue from the Sensors and Biosensors Group Barcelona (Department of Chemistry, Universitat Autònoma de Barcelona) registers RedOx processes taking place at the surface of differently modified working electrodes $[11,21]$. Unlike potentiometric sensors this type of measurements can provide information on non-ionic substances in the media which can be reduced/oxidized under experimental conditions.

So far, comparability studies have been performed based on the two commercially available systems $[22,23]$ or proving the interlab comparability of the Insent taste sensing system [24]. Since the sensor systems applied for pharmaceutical analyses are based on different principles and may have different functionality, it seems timely to perform independent comparison of the several above mentioned e-tongues in the framework of an interlaboratory experiment to reveal possible differences and special aspects associated with each of the systems. The purpose of this study was an independent comparison of six different multisensor systems applied to the same set of samples. In order to eliminate subjective factors all the raw data from the systems, obtained in blind conditions, were processed in centralized way with the same algorithms and approaches.

\section{Materials and methods}

\subsection{Materials}

Caffeine Citrate (Fagron, Barsbuettel, Germany), maltodextrin (Kleptose ${ }^{\circledR}$ linecaps 17, Roquette Frères, France), citric acid (J.T.
Baker, Griesheim, Germany), saccharin sodium (Caesar \& Loretz, Hilden, Germany), lactose monohydrate (granuLac ${ }^{\circledR} 140$, Meggle Pharma, Wasserburg, Germany) were used to prepare the samples. Potassium Chloride (Gruessing, Filsum, Germany), tartaric acid (AppliChem, Darmstadt, Germany), hydrochloric acid (Merck, Darmstadt, Germany), potassium hydroxide (Gruessing, Filsum, Germany) and absolute ethanol (VWR international, Darmstadt Germany) were used to prepare the washing solutions for the Insent e-tongue. Potassium chloride, used at the University of Barcelona, was purchased from Merck KGaA (Darmstadt, Germany) and the solutions were prepared using deionised water from a Milli-Q system (Millipore, Billerica, MA, USA). Purified water was produced by appropriate methods, such as reverse osmosis at the Heinrich-Heine-University of Duesseldorf (Germany) and at Warsaw University of Technology, Poland (Millipore Elix3) and double distillation with GFL-2101 system (GFL, Germany) at St. Petersburg University.

\subsection{Sample preparation}

To perform a concentration series based on caffeine citrate, $0.386 \mathrm{~g}$ caffeine citrate was dissolved in $100.0 \mathrm{ml}$ of purified water, resulting in a $10 \mathrm{mM}$ stock solution. Based on this stock solution, a serial dilution series (10-1 mM, then 1-0.1 mM, then 0.01-0.001 $\mathrm{mM}$ ) were performed for the e-tongues based on potentiometric signal acquisition. For the voltammetric e-tongue sensitivity of the sensors was proven by measuring a concentration series including $0.38 \mathrm{mM}, 0.57 \mathrm{mM}, 0.95 \mathrm{mM}, 1.88 \mathrm{mM}, 3.68 \mathrm{mM}$ and $7.02 \mathrm{mM}$ in an aqueous $\mathrm{KCl}$ solution $(10 \mathrm{mM})$.

Samples for the taste-masking experiment were prepared according to Table 1 . The substances were precisely weighed into $100 \mathrm{ml}$ flasks and shipped to all research groups involved in the study. Each sample was then diluted at site with $100.0 \mathrm{ml}$ of purified water according to a shared protocol and measured under blind conditions. The $\mathrm{pH}$ values of samples $1-4,6,8,9$ were around 2.5 and for samples 5 and 7 (containing neither caffeine citrate nor citric acid) the $\mathrm{pH}$ was around 5.5.

\subsection{Description of the applied electronic tongues and measurement protocols}

\subsubsection{TS-5000Z (Insent, Inc., Atsugi-Shi, Japan) (Fig. 1a)}

The TS-5000Z e-tongue system (abbreviation: I) was equipped with the commercially available sensors SB2ACO, SB2ANO and SB2BT0 (dedicated to bitter cationic substances), SB2AAE (dedicated to umami), SB2CT0 (dedicated to saltiness) and SB2CA0 (dedicated to sourness), SB2C00 (dedicated to bitter anionic substances) and SB2AE1 (dedicated to astringent substances) and $\mathrm{Ag} / \mathrm{AgCl}$-reference electrode. The measurement followed the standard procedure as described by Woertz et al. [22,25], using the recommended measurement setup $A B C A B C(A, B$, and $C$ are representatives of sample beakers). Prior to each measurement, a sensor check was performed and only proper working sensors were applied further. The washing steps were conducted in the recommended washing solutions (-)-solution: $100 \mathrm{mM}$ hydrochloric acid diluted with ethanol $(30 \%(\mathrm{w} / \mathrm{w}))$ for negatively charged sensors, (+)-solution: $100 \mathrm{mM}$ potassium chloride and $10 \mathrm{mM}$ potassium hydroxide in ethanol $(30 \%(\mathrm{w} / \mathrm{w}))$ as well as in the standard solution $(0.3 \mathrm{mM}$ tartaric acid and $30 \mathrm{mM}$ potassium chloride in distilled water). The whole measurement procedure was carried out 4 times in a row. Regarding the conditioning of the sensors, only the results of the last three runs were considered for evaluation.

Measurement of the samples followed the procedure described by Pein et al. [24], starting with the bitter sensors SB2ACO, SB2ANO and SB2BT0 in the first cycle, followed by a second run with the other 5 sensors. 
Table 1

Composition and labelling of the analyzed samples.

\begin{tabular}{|c|c|c|c|c|c|c|c|c|c|c|}
\hline \multirow[b]{2}{*}{ Substance } & \multirow[b]{2}{*}{ Amount per sample } & \multicolumn{9}{|c|}{ Sample $\mathrm{n}^{\circ}$} \\
\hline & & 1 & 2 & 3 & 4 & 5 & 6 & 7 & 8 & 9 \\
\hline Caffeine citrate & $0.34 \mathrm{~g}$ & $\mathrm{x}$ & $\mathrm{x}$ & & $\mathrm{x}$ & & $\mathrm{x}$ & & $\mathrm{x}$ & \\
\hline Lactose monohydrate & $2.12 \mathrm{~g}$ & $\mathrm{x}$ & $\mathrm{x}$ & $\mathrm{x}$ & $\mathrm{x}$ & $\mathrm{x}$ & $\mathrm{x}$ & $\mathrm{x}$ & $\mathrm{x}$ & $\mathrm{x}$ \\
\hline Maltodextrine & $0.85 \mathrm{~g}$ & & & & & & $\mathrm{x}$ & $\mathrm{x}$ & $\mathrm{x}$ & $\mathrm{x}$ \\
\hline Saccharin sodium & $0.0085 \mathrm{~g}$ & & & & $\mathrm{x}$ & $\mathrm{x}$ & & & $\mathrm{x}$ & $\mathrm{x}$ \\
\hline Citric acid & $0.17 \mathrm{~g}$ & & $\mathrm{x}$ & $\mathrm{x}$ & & & & & $\mathrm{x}$ & $\mathrm{x}$ \\
\hline
\end{tabular}

a)

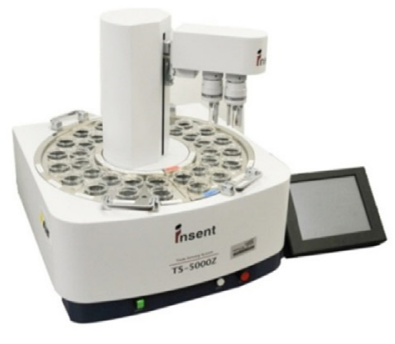

c)

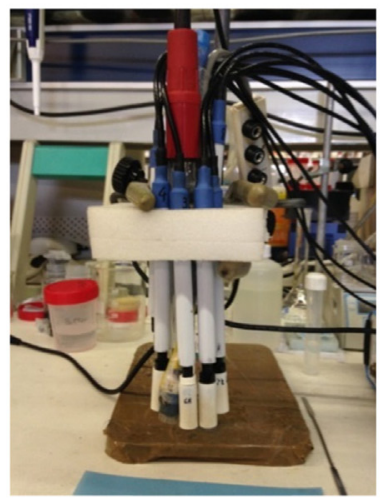

e)

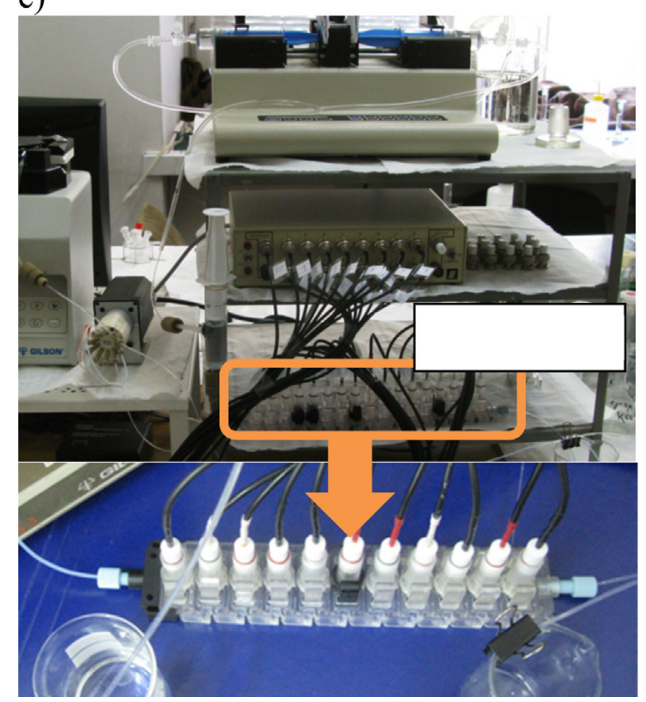

b)

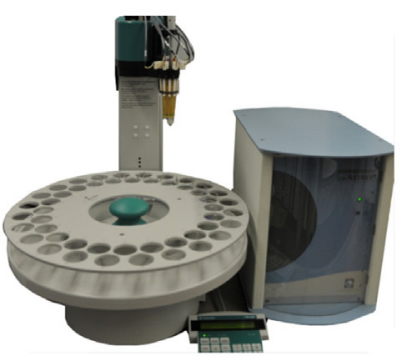

d)

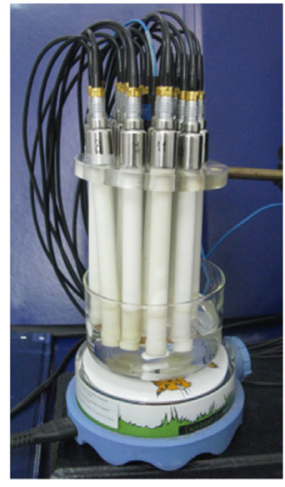

f)

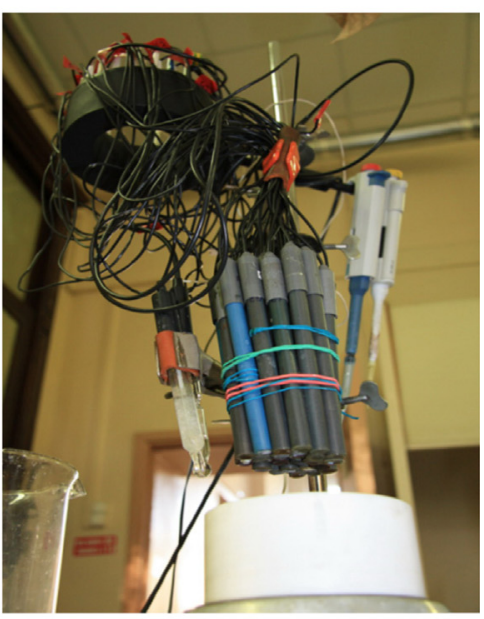

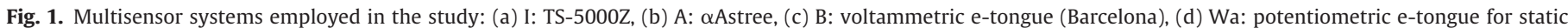
measurements (Warsaw), (e) Wb: potentiometric e-tongue for FIA measurements (Warsaw), (f) SP: potentiometric e-tongue (St. Petersburg).

\subsection{2. $\alpha$ Astree (AlphaMOS, Toulouse, France) (Fig. 1b)}

The $\alpha$ Astree e-tongue system (abbreviation: A) was equipped with the sensor set for pharmaceutical applications (sensor set \#2), an $\mathrm{Ag} / \mathrm{AgCl}$ reference electrode and a stirrer. Applied cross-selective sensors $\mathrm{ZZ}, \mathrm{AB}, \mathrm{GA}, \mathrm{BB}, \mathrm{CA}, \mathrm{DA}$ and JE, were stored dry before the starting procedure. To prove the operational qualification, the steps 'conditioning', 'calibration' and 'diagnostic' were carried out prior to experiment sequences. 
According to the qualification protocol [26], the experiment sequence was built according to AABBCC order ( $A, B$, and $C$ represent different sample concentrations). To perform the measurement, sensors were dipped into a sample beaker and each sample was analyzed over a period of 120 s subsequently eight times. After this procedure the sensors were dipped into a washing beaker, three times $10 \mathrm{~s}$, before the next sample was analyzed.

\subsubsection{Barcelona voltammetric e-tongue (Fig. 1c)}

The voltammetric e-tongue from the working group in Barcelona (abbreviation: B) was formed by an array of 6 sensors, plus a reference double junction $\mathrm{Ag} / \mathrm{AgCl}$ electrode (Thermo Orion 900200, Beverly, MA) and a commercial Pt counter electrode (Model 52-67, Crison Instruments, Barcelona, Spain). Working electrodes were metallic electrodes and bulk modified graphite epoxy composites: two metallic Au and Pt electrodes plus four composite electrodes, one unmodified electrode and three composite electrodes modified with $\mathrm{Cu}$ nanoparticles, Pt nanoparticles and $\mathrm{Co}(\mathrm{II})$ phtalocyanine, respectively.

Electrochemical measurements were performed at room temperature $\left(25^{\circ} \mathrm{C}\right)$, using a 6-channel AUTOLAB PGSTAT20 (Ecochemie, Netherlands) controlled with GPES Multichannel 4.7 software package. A voltammogram was recorded from $-1.0 \mathrm{~V}$ to $+1.75 \mathrm{~V}$ vs. $\mathrm{Ag} / \mathrm{AgCl}$ with a step potential of $9 \mathrm{mV}$ a scan rate of $100 \mathrm{mV} \mathrm{s}^{-1}$ and an amplitude of $0.02505 \mathrm{~V}$. Moreover, in order to get stable voltammetric responses, ensuring reproducible signals from the ET array along the whole experiment, two extra precautions were taken. Firstly, electrodes were cycled in buffer solution after its polishing and beginning of samples measurements. Secondly, an electrochemical cleaning stage was carried out between each measurement to prevent accumulative effect of impurities on the working electrode surfaces. For this purpose a conditioning potential of $-1.50 \mathrm{~V}$ was applied during $45 \mathrm{~s}$ in a cell containing $30 \mathrm{ml}$ of distilled water.

\subsubsection{Potentiometric e-tongue for static (batch) measurements (Warsaw) (Fig. 1d)}

The sensor array of the Warsaw batch e-tongue (abbreviation Wa) was based on 13 electrodes equipped with polymeric membranes with various electroactive additives. There were sensors exhibiting general selectivity to lipophilic cations, general selectivity to lipophilic anions as well as sensors selective to various organic species with carboxylic or amine group; as the reference standard double junction $\mathrm{Ag} / \mathrm{AgCl}$ electrode was applied. Prior to measurements of pharmaceutical samples, all sensors were qualified on the basis of their response repeatability and appropriate sensitivity. Sensor check was performed each day of the measurements, moreover before the signal acquisition in every sample sensor response was stabilized in standard solution. Each sample measurement time was $4 \mathrm{~min}$ with signal acquisition every $3 \mathrm{~s}$. Final output of the array was calculated as a mean value of 10 last readings for each sensor.

\subsubsection{Potentiometric e-tongue for FIA measurements (Warsaw) (Fig. 1e)}

The sensor array of the Warsaw FIA e-tongue (abbreviation Wb) was based on 14 miniaturized electrodes equipped with various polymeric membranes to obtain sensors exhibiting general selectivity to ionic lipophilic species and organic species with carboxylic or amine group. The sensors were mounted in a flow-through cell in a sequence together with miniaturized double-junction reference electrode. The design of the modular flow system is a subject of a polish patent application [27] and was previously reported [28-30]. The fluid system was composed of: a two position ten port Cheminert ${ }^{\circledR}$ valve (Vici, USA), a sample loop (200 ul), a peristaltic pump (Minipuls 3, Gilson, France) used to fill the sample loop, and a syringe pump (KDS Model 200 Series, Holliston, USA) used to introduce the carrier solution into the flow-through sensor array. All elements were connected together using low-pressure connectors; additionally, air bubble catcher to remove air from carrier solution was applied [31]. Sensor qualification, sensor check, signal stabilization were applied in the form of the same procedure as in the case of Warsaw e-tongue for batch measurements. Each sample was injected in triplicate to the carrier solution $\left(10^{-3} \mathrm{M} \mathrm{NaNO}_{3}\right)$; the mean of the three peak heights served as a sensor response for further data analysis.

\subsubsection{Potentiometric multisensor system of 23 electrodes (St. Petersburg) (Fig. 1f)}

The potentiometric multisensor system based on 23 crosssensitive electrodes (abbreviation: SP) was employed during the experiments in St. Petersburg. These were nine cation-sensitive sensors, 11 anion-sensitive sensors, two chalcogenide glasses with pronounced red/ox sensitivity and standard glass pH-sensor ESL43-07 (ZIP, Gomel, Belorussia). All potentiometric measurements were performed against $\mathrm{Ag} / \mathrm{AgCl}$ reference electrode with custom made 32-channel digital $\mathrm{mV}$-meter connected to a PC through USB interface. Precision in potential measurements was $0.1 \mathrm{mV}$. Typical measurement time was $3 \mathrm{~min}$ for each sample. After the measurements, sensor array was washed several times with distilled water to bring the potentials of the sensors to initial readings.

\subsection{Data analysis}

All the data obtained from different e-tongues were evaluated with Excel, Simca-P+ 12.0.1 and R software. The resulting sizes of the data matrices were:

- potentiometric e-tongue for batch measurements (Warsaw): $45 \times 13$ (9 sample types, all measured in 5 repetitions by 13 sensors)

- potentiometric e-tongue for FIA measurements (Warsaw): $45 \times 14$ ( 9 sample types, all measured in 5 repetitions by 14 sensors)

- potentiometric e-tongue (St. Petersburg): $45 \times 23$ (9 sample types, all measured in 5 repetitions by 23 sensors)

- potentiometric e-tongue (TS-5000Z, Insent Inc.): $36 \times 8$ (9 sample types, all measured in 4 repetitions by 8 sensors)

- ISFET based e-tongue ( $\alpha$ Astree, AlphaMOS): $45 \times 7$ (9 sample types, all measured in 5 repetitions by 7 sensors)

- voltammetric e-tongue (Barcelona): $27 \times 1242$ (9 sample types, all measured in 3 repetitions by 6 sensors, 207 data points for each sensor).

Comparison of the results obtained from the different e-tongues was performed based on principal component analysis (PCA), on the calculation of $\mathrm{RV}^{\prime}$ matrix correlation coefficients and Tucker's congruency coefficients. PCA is the method of data dimensionality reduction and visualization of the hidden data structure. It is widely employed in numerous studies nowadays and extended details can be found in [32]. One of the main outcomes of PCA analysis are so called score plots, visualizing similarity of the studied samples in $2 \mathrm{D}$ plots. Both $\mathrm{RV}^{\prime}$ and Tucker's $\phi$ are intended for assessment of similarity between two data sets sharing the same row mode (i.e., obtained for the same sets of samples). The $\mathrm{RV}^{\prime}$ coefficient can be calculated as follows [33]:

$\operatorname{RV}^{\prime}(X, Y)=\frac{\operatorname{tr}\left(\overline{\mathrm{XX}}^{\mathrm{T}} \overline{\mathrm{YY}}^{\mathrm{T}}\right)}{\sqrt{\operatorname{tr}\left[\left(\overline{\mathrm{XX}}^{\mathrm{T}}\right)^{2} \operatorname{tr}\left[\left(\overline{\mathrm{YY}}^{\mathrm{T}}\right)^{2}\right]\right]}}$, where 
$\overline{\mathrm{XX}}^{\mathrm{T}}=\left[\mathrm{XX}^{\mathrm{T}}-\operatorname{diag}\left(\mathrm{XX}^{\mathrm{T}}\right)\right]$

Here $X$ and $Y$ are the response matrices obtained from two different analytical instruments (different e-tongues) for the same set of sensors. The $\mathrm{RV}^{\prime}$ coefficient can be interpreted as analogous to Pearson's correlation coefficient and it can have values in the range between -1 and +1 (negative and positive correlation correspondingly) [33]. In order to estimate the similarity between the latent variables for two data sets, e.g., in PCA, Tucker's congruence coefficient can be employed [34]:

$\phi=\frac{\operatorname{tr}\left(T_{1} T_{2}^{T}\right)}{\sqrt{\operatorname{tr}\left(T_{1} T_{1}^{T} \operatorname{tr}\left(T_{2} T_{2}^{T}\right)\right)}}$

where $T_{1}$ and $T_{2}$ are score matrices obtained by PCA for two data sets with the same number of rows. Tucker's coefficient may vary from -1 to +1 .

\section{Results and discussions}

\subsection{Evaluation of the dilution series}

As a first stage of research, the responses of different multisensor systems in caffeine citrate solutions of varying concentration were analyzed to ensure that all the e-tongues under study possess certain sensitivity towards the investigated active pharmaceutical ingredient caffeine citrate. In Fig. 2, responses of four typical sensors from each system are shown except the voltammetric e-tongue for which the response of only one sensor is given for the sake of better visualization on a single plot.

It can be seen that the sensors in all the studied systems have pronounced sensitivity towards the components of caffeine citrate solutions. In case of Insent and potentiometric tongues from St. Petersburg and Warsaw, this sensitivity obviously can be explained by response to citrate anions and caffeine cations (negative and positive sloped curves correspondingly). The same applies for the ISFET based $\alpha$ Astree. In case of voltammetric system, Fig. $2 c$ shows the calibration curve for the epoxy graphite sensor. Although the other sensors provide relevant information about the compounds that have redox activity, the epoxy graphite sensor together with the Au are the ones that have higher sensitivity towards the caffeine citrate.

Unfortunately direct comparison of distinct sensitivity parameters is not possible for all studied systems, since they are based on different analytical platforms (ISFETs, potentiometric sensors, voltammetry) and moreover they have different measurement methodology. For example TS-5000Z requires reference solution measurements between all assessments and response is calculated against the sensor readings in this solution, while potentiometric systems from St. Petersburg and Warsaw (e-tongue for batch measurements) are using sensor responses obtained directly from the measurements against reference electrode. On the other hand, in the case of Warsaw FIA e-tongue the output is calculated versus potentials obtained in the carrier solution because peak heights are considered. For the $\alpha$ Astree, an individual factor, which is assigned but hidden in the software, is multiplied with each of the sensor responses.

\subsection{Evaluation of $R V^{\prime}$ matrix correlation coefficients and Tucker's congruency coefficients}

Besides the often used principal component analysis, another possible way to compare multivariate data obtained for the same set of samples is to calculate corresponding matrix correlation coefficients. For this purpose RV' coefficients for raw data and Tucker's congruency coefficients for PCA scores were calculated (Table 2) based on the data from each e-tongue obtained from the nine samples shown in Table 1 . The more similar the data matrices from two methods the closer corresponding $\mathrm{RV}^{\prime}$ value to \pm 1 , otherwise it tends to zero. The same holds for Tucker's $\phi$.

Summarized in Table 2, there are the $\mathrm{RV}^{\prime}$ coefficients (fields marked in grey) and the Tucker's congruency coefficients (fields marked in light grey); both sets reveal that certain similarity exists between all studied pairs of data sets from e-tongue systems. The e-tongues with many variables (St. Petersburg and Barcelona) are generally showing higher RVí values than those with smaller number of variables (columns in the data matrix). Two most similar in this metric data sets are originating from St. Petersburg and Warsaw (batch version) $\left(\mathrm{RV}^{\prime}=0.992\right)$. This is well understood taking into account the same analytical platform (potentiometry) and wide similarities in sensor compositions (polymeric plasticized membranes) of these two systems. Two most dissimilar systems in $\mathrm{RV}^{\prime}$ metrics are Warsaw FIA version $(\mathrm{Wb})$ and $\alpha$ Astree $(\mathrm{A})$, which can be attributed to the higher number of sensors in the system from Warsaw, different measuring protocol (flow cell) and different sensor technology (ISFET in $\alpha$ Astree against potentiometric platform). Situation changes when considering Tucker's congruency coefficients (Table 2, highlighted in lighter grey), their values imply much smaller number of correlated data sets in terms of variance structure. Now the two most similar data sets are SP and $\mathrm{Wb}(\phi=0.779)$, and the most dissimilar are I and $\mathrm{Wb}(\phi=0.005)$. Tucker's congruency coefficients can be considered as more appropriate metrics for this particular task, since these values assess the variance structure within the data and they are more relevant for the comparison of the techniques involving different sensor types and measuring protocols.

According to the results of application of different mathematical methods, all studied e-tongue systems are more or less comparable in terms of their ability to capture the variance in the data resulting from the studied samples.

\subsection{Evaluation of the taste-masked samples}

For this particular experiment, different taste-masking agents were solely or in combination added to a basic formulation containing caffeine citrate and lactose (Table 1). Applicability of different sensor arrays to detect the masking-ability of citric acid as counterpart within caffeine citrate (sample 3) and the highly potent sweetener saccharin sodium (sample 5) was the purpose of evaluation. Maltodextrin grades have recently been discussed as possible taste-masking agent within a preformulation study utilizing electronic tongues [35]. Thus, the most promising candidate was also included in some formulations (samples 6-9). Moreover, it should be investigated, if the sensor arrays can differentiate precisely between drug containing formulations (samples 1, 2, 4, 6, 8; Table 1) and corresponding placebo formulations. By mixing all taste-masking agents (sample 9), the taste-masking agent with the highest impact on the sensor responses should be defined. From the human sensory point of view, this was expected for saccharin sodium, since it provides a very sweet taste, but sometimes even described as metallic [36].

\subsubsection{Comparison of the data obtained with the different e-tongues}

PCA was employed to compare the recognition capabilities of the studied electronic tongues. PCA results are usually presented as the score plots. The idea of these plots is to visualize the difference/similarity in the measured samples. As an example Fig. 3 can be considered. Each point shown in the plot is the particular sample (measurement results) in a principal component (PC) space. Those points (samples) which are located close to each other in a score plot are similar (due to similar corresponding multisensor 
a)

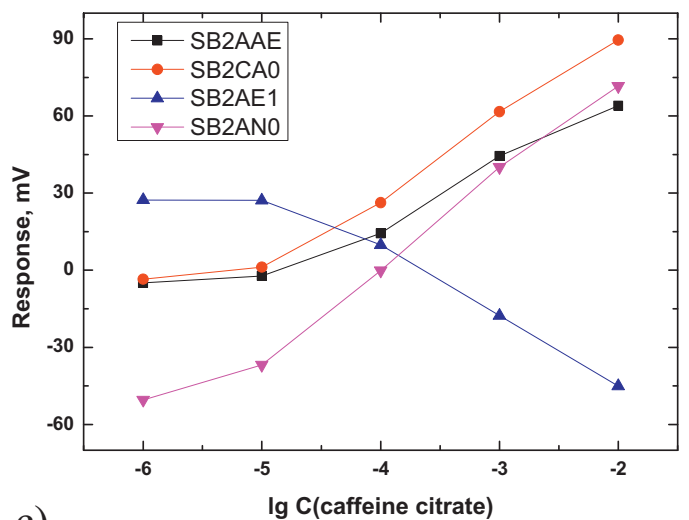

c)

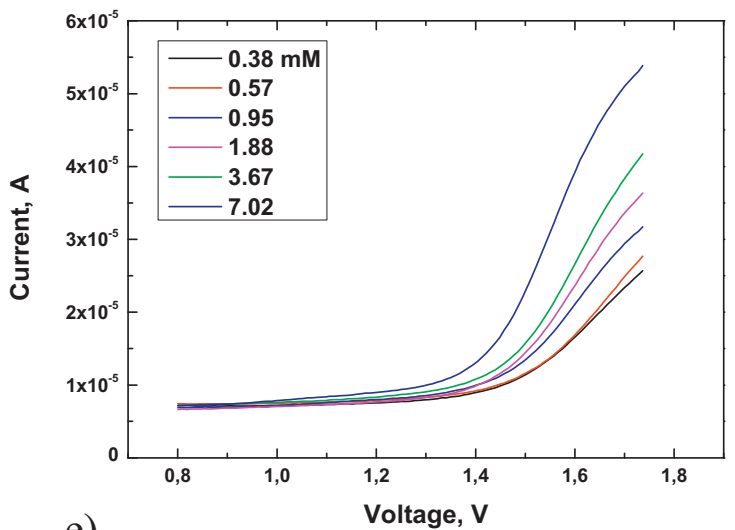

e)

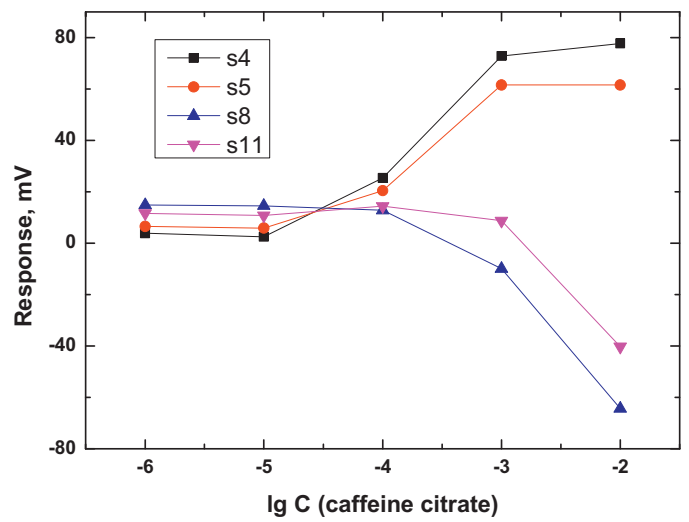

b)

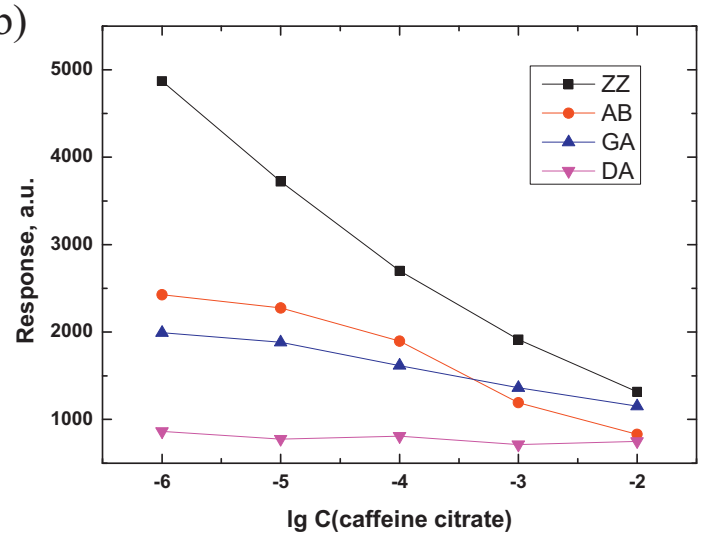

d)

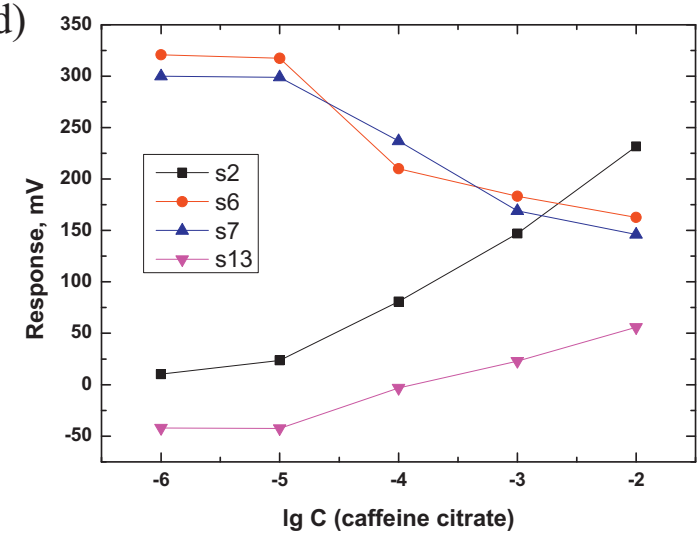

f)

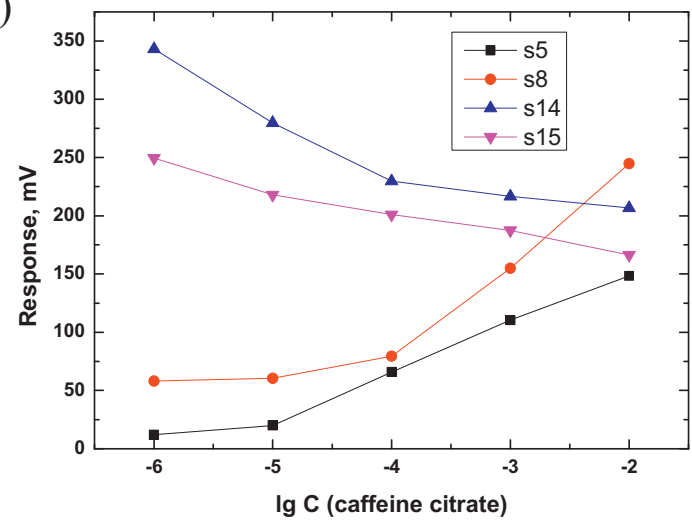

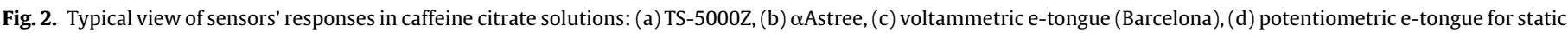
measurements (Warsaw), (e) potentiometric e-tongue for FIA measurements (Warsaw), (f) potentiometric e-tongue (St. Petersburg).

Table 2

RVí matrix correlation coefficients displayed in grey and Tucker’s congruency coefficients $(\phi)$ displayed in light grey.

\begin{tabular}{|l|l|l|l|r|r|r|}
\hline & I & \multicolumn{1}{l|}{ A } & \multicolumn{1}{l|}{ B } & Wa & Wb & \multicolumn{1}{l|}{ SP } \\
\hline I & - & 0.753 & 0.873 & 0.772 & 0.820 & 0.811 \\
\hline A & -0.096 & - & 0.860 & 0.846 & 0.588 & 0.808 \\
\hline B & 0.098 & 0.091 & - & 0.969 & 0.897 & 0.980 \\
\hline Wa & 0.327 & 0.287 & 0.297 & - & 0.863 & 0.992 \\
\hline Wb & 0.005 & 0.742 & -0.622 & 0.373 & - & 0.920 \\
\hline SP & 0.256 & -0.739 & -0.614 & 0.091 & 0.779 & - \\
\hline
\end{tabular}

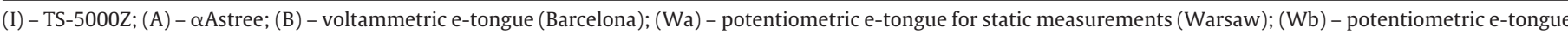
for FIA measurements (Warsaw); (SP) - potentiometric e-tongue (St. Petersburg). 
a)

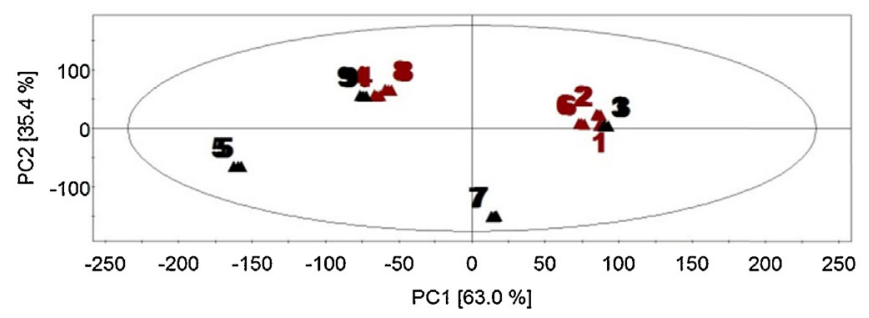

c)

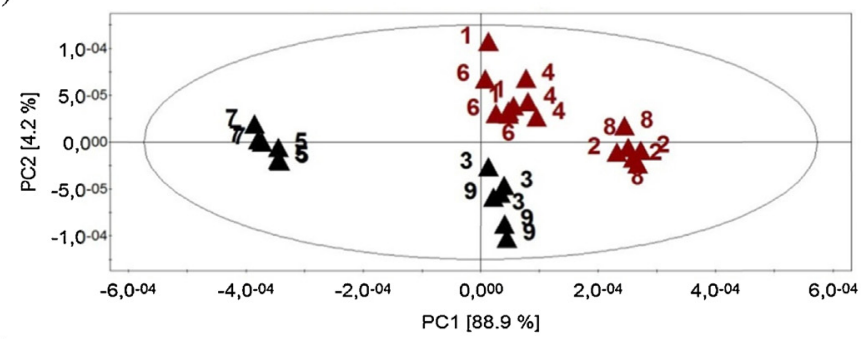

e)

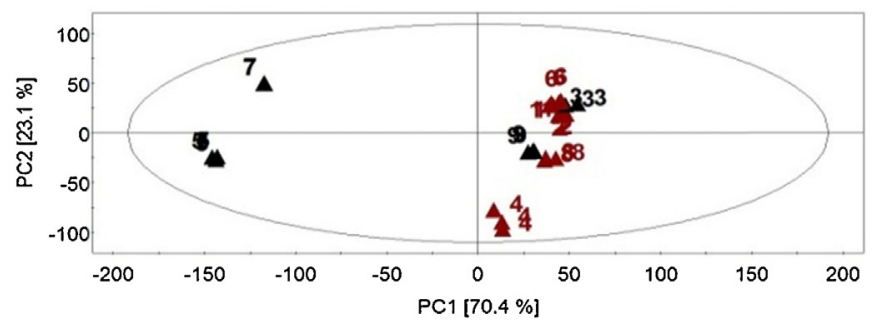

b)

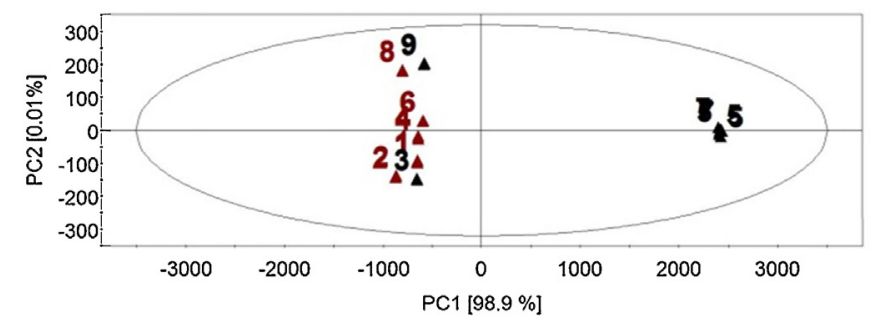

d)

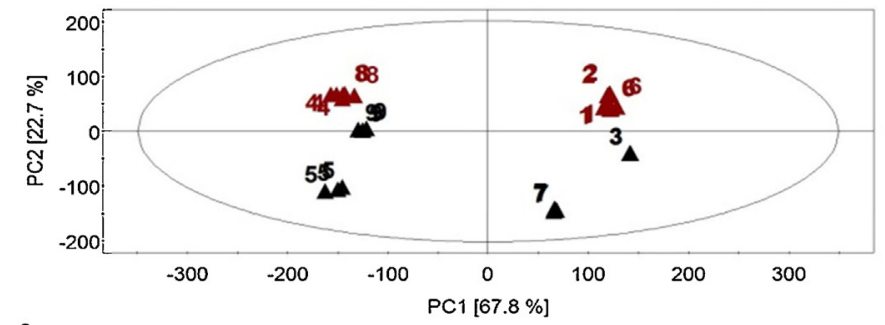

f)

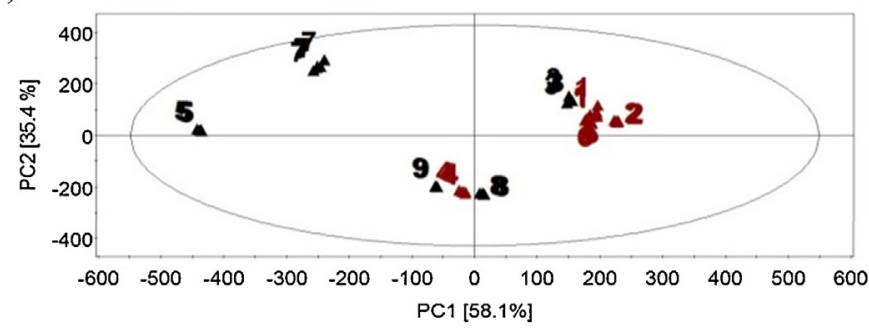

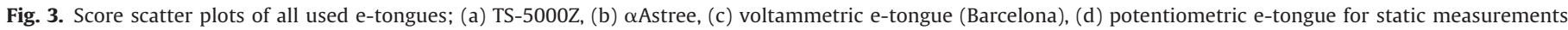

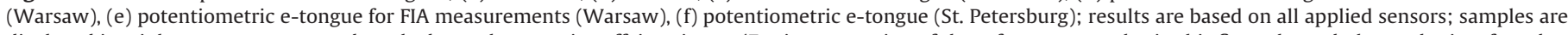

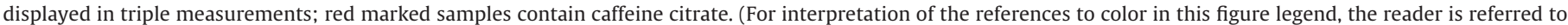
the web version of this article.)

system responses). In the brackets at the axis captions the amount the variance in the data explained by each PC is shown. These numbers must be also taken into account when judging on recognition capabilities of the systems.

With regard to this, placebo samples 5 (containing saccharin sodium) and 7 (containing maltodextrin) were detected to be dissimilar from the other samples, when measured with the Warsaw "FIA" e-tongue, the $\alpha$ Astree, the SP e-tongue and the Barcelona voltammetric e-tongue (Fig. 3). These distinctions are most likely dedicated to the differing $\mathrm{pH}$ value of samples 5 and 7 ( $\mathrm{pH} \mathrm{5.5)}$ compared to the other samples ( $\mathrm{pH} 2.5$ )

For each potentiometric based sensor array the expected influence of saccharin sodium could be confirmed: the according sweetener-free and sweetener-containing samples were discriminated either along the $x$-axes (Insent (Fig. 3a) and Warsaw "Batch" e-tongue (Fig. 3d)) or the $y$-axes (SP e-tongue (Fig. 3f) and Warsaw "FIA" e-tongue (Fig. 3e)). Interestingly, distinction between caffeine citrate containing samples (samples $1,2,4,6,8$ ) and drug free samples along the first principal component axis (along the direction of the most variability in the data) was made by none of the electronic tongues. However, Barcelona e-tongue and Warsaw "batch" e-tongue allow for discrimination between drug containing samples (red) and placebo samples (black) - certain clustering is visible along the second PC. It is rather that caffeine citrate was detected most likely as citric acid and thus, samples containing citric acid and/or caffeine citrate and samples without any of the two ingredients were significantly discriminated along the major component by the voltammetric sensors of the Barcelona e-tongue as well as the $\alpha$ Astree, the Warsaw "FIA" e-tongue and the SP e-tongue.
When performing PCA excluding samples 5 and 7 and thus those samples, which neither contained caffeine citrate nor citric acid, and showed a differing $\mathrm{pH}$ value, the four potentiometric working e-tongues provided for pronounced discrimination between samples containing saccharin sodium (samples $4,8,9$ ) and those without saccharin sodium (samples $1,2,3,6$ ) (Fig. 4). It is also noteworthy that the potentiometric systems from St. Petersburg (Fig. 4f) and Warsaw (Fig. 4d: "Batch" e-tongue) provide similar recognition ability, resulting in corresponding scatter plots. The St. Petersburg's e-tongue was able to discriminate the samples with caffeine citrate from those without the API along the second principal component (Fig. 4f, PC 2 describing $14.4 \%$ of the variance). The samples 4,8 and 9 were located somewhat closer to each other based on the Warsaw "Batch" results (Fig. 4d, PC2 describing $9.8 \%$ of the total variance). This strong analogy in the performance of two systems are due to wide similarities in measuring protocol and sensors as it was already mentioned in the discussion of Tucker's coefficients (Section 3.2). The slight differences might be due to the increased number of sensors (23 sensors vs. 14 sensors), which are applied at the St. Petersburg e-tongue. Samples 2 and 8, which contained caffeine citrate plus citric acid, were detected to be significantly different from the other samples from the voltammetric Barcelona e-tongue (along PC 1 with 64.6\%) and to a little less degree also from the $\alpha$ Astree (along PC 2 with $31.8 \%$ ). For the voltammetric tongue, this is in a good agreement with the measurement principle, which allows for detecting substances that can be reduced or oxidized best. For the $\alpha$ Astree, this result might be due to the acidic character of these particular samples. Studying Figs. 3 and 4 also allows noticing that voltammetric e-tongue provides a little less reproducibility in replicated measurements compared to other 
a)

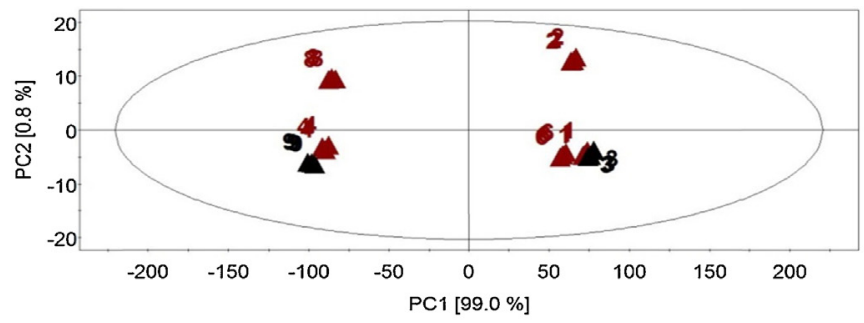

c)

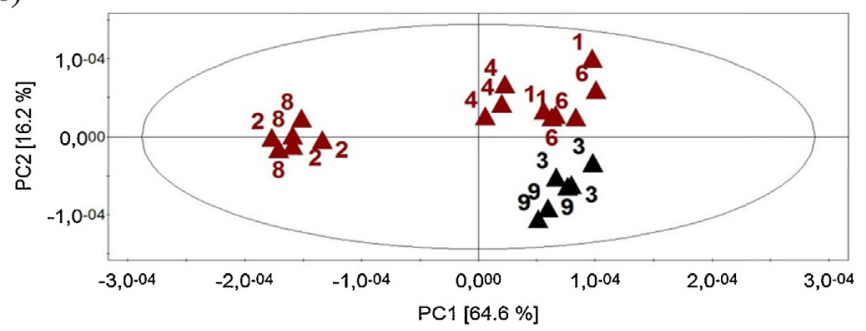

e)

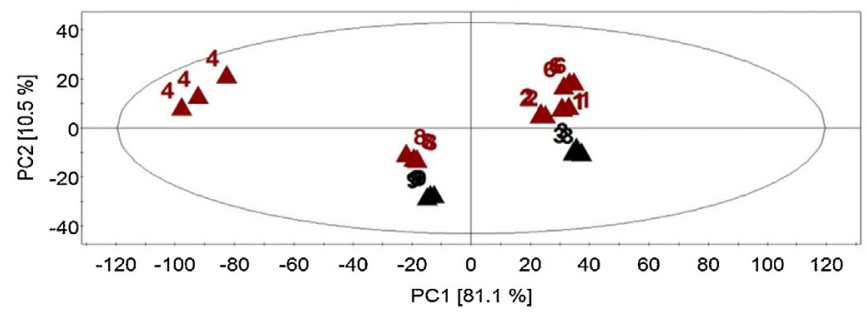

b)

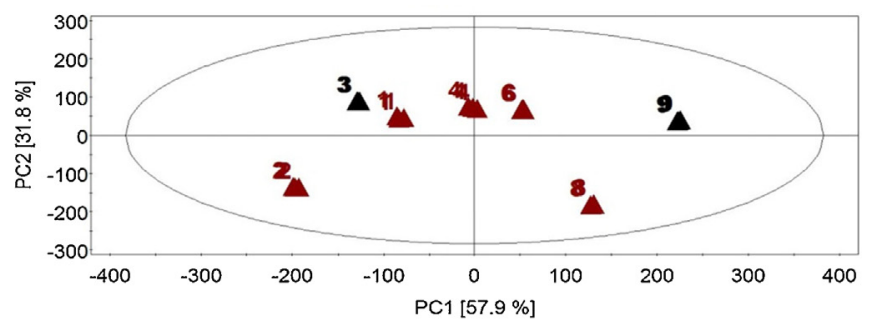

d)

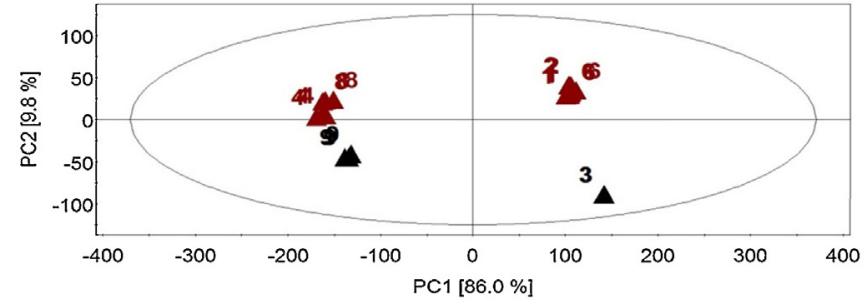

f)

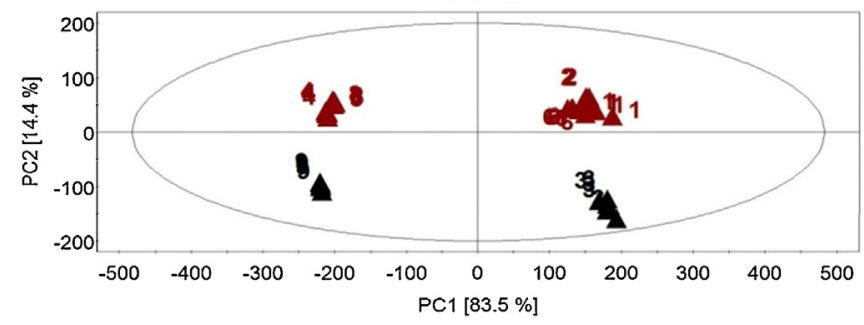

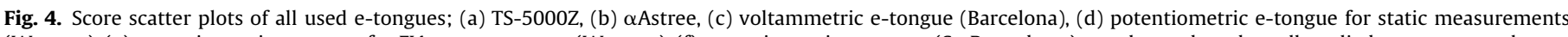

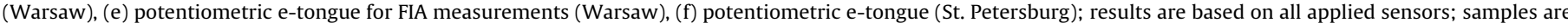

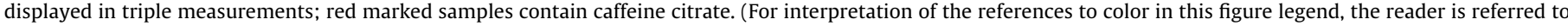
the web version of this article.)

Table 3

Variance structure in terms of explained variance in PCA (Fig. 4).

\begin{tabular}{lllllll}
\hline$\%$ & PC1 & PC2 & PC3 & PC4 & PC5 & PC6 \\
\hline I & 99 & 1 & 0 & 0 & 0 & 0 \\
A & 58 & 32 & 9 & 1 & 0 & 0 \\
B & 65 & 16 & 11 & 4 & 2 & 1 \\
Wa & 86 & 10 & 2 & 1 & 0 & 0 \\
Wb & 81 & 11 & 6 & 1 & 1 & 0 \\
SP & 83 & 14 & 1 & 1 & 0 & 0 \\
\hline
\end{tabular}

(I) - TS-5000Z; (A) - $\alpha$ Astree; (B) - voltammetric e-tongue (Barcelona); (Wa) potentiometric e-tongue for static measurements (Warsaw); ( $\mathrm{Wb}$ ) - potentiometric e-tongue for FIA measurements (Warsaw); (SP) - potentiometric e-tongue (St. Petersburg)

studied systems, since corresponding points in the same samples are scattered. This scatter is originated from electrical noise at high potential of operation, where oxidation signal of caffeine is distorted by simultaneous oxidation of water. This problem can be avoided by optimizing sensor compositions in order to obtain more pronounced catalytic response towards caffeine, e.g., incorporating polypyrrole [37] or graphene [38] as electrode modifiers.

Table 3 summarizes the percentage of explained variance in PCA decomposition for each particular data set (related to Fig. 4). The study of variance distribution along the PCs also allows assessing data similarity. In terms of this distribution, all potentiometric systems except Insent are pretty similar to each other. Voltammetric e-tongue shows the smallest variance explained by the first two PCs and it takes six PCs to achieve the total explained variance of $98 \%$, but it is also the system with high dimensionality. For Insent two first PCs give $100 \%$. The latter result suggests that Insent system in this study does not provide for recognition of the samples along the PC2 component, since the overall variance amount for PC2 is below $1 \%$. It is also noticable, that the other three potentiometric systems (SP e-tongue, $\mathrm{Wa}$ and $\mathrm{Wb}$ ) provide very similar variability structure (almost the same amount of variance captured in the first two PCs).

\section{Conclusion}

Machine assessment of taste parameters in pharmaceutics appears to be an attractive option for traditional sensory panels. E-Tongue technology can provide for fast taste profiling and it does not suffer from fatigue effect. This study compared for the first time the performance of six different e-tongues (both, commercially available and at R\&D stage) in the framework of a blind experiment dedicated to the analysis of several pharmaceutical formulations. In order to obtain unbiased evaluation of systems' performance, samples were identical for all applied e-tongues and data analysis was performed in a centralized way by employing the same algorithms and procedures for all the systems. Comparison of data sets obtained from different e-tongues was performed by using matrix correlation coefficients, Tucker's coefficients and principal component analysis. The obtained results revealed that laboratory prototypes of potentiometric e-tongues recognized the studied pharmaceutical formulations in a rather similar manner, while the voltammetric system led to a different recognition picture. Due to its particular measurement principle this system shows sensitivity towards substances, which are not ionized in solution but can be reduced or oxidized and it can be valuable for various applications. In certain cases the use of bigger number of sensors 
in multisensor array (St. Petersburg system with 23 sensors) provides for more distinct clustering in PCA plots. It is noteworthy that only two systems: voltammetric e-tongue and Warsaw "batch" etongue, can differentiate samples containing caffeine citrate from API-free samples when processing all samples simultaneously and this can be beneficial for studying of taste-masking effects and drug-release kinetics.

It must be pointed out that all these findings are so far based on the investigation of a limited set of pharmaceutical samples and for more general conclusion the studied e-tongues should be further evaluated applying different APIs and pharmaceutical dosage forms. For sure these ongoing comparative studies may be of great help for potential users in the pharmaceutical field.

\section{Acknowledgements}

University of Duesseldorf thanks AlphaMOS for providing the taste sensing system $\alpha$ Astree. M. del Valle thanks the ICREA Academia program 2010. The work of Russian participants was partially financially supported by Government of Russian Federation, Grant 074-U01. The work performed at the University of Warsaw has been financially supported within a framework of "Diamond Grant" programme and OPUS project "Sensor arrays for the study of the release process of active pharmaceutical ingredients and excipients from pharmaceuticals" UMO-2013/09/B/ST4/00957.

\section{References}

[1] K. Toko, Taste sensor with global selectivity, Mater. Sci. Eng. C 4 (1996) 69-82.

[2] K. Toko, A taste sensor, Meas. Sci. Technol. 9 (1998) 1919-1936.

[3] Y. Miyanaga, A. Tanigake, T. Nakamura, Y. Kobayashi, H. Ikezaki, A. Taniguchi, K. Matsuyama, T. Uchida, Prediction of the bitterness of single, binary- and multiple-component amino acid solutions using a taste sensor, Int. J. Pharm. 248 (2002) 207-218.

[4] Y. Kobayashi, M. Habara, H. Ikezazki, R. Chen, Y. Naito, K. Toko, Advanced taste sensors based on artificial lipids with global selectivity to basic taste qualities and high correlation to sensory scores, Sensors 10 (2010) 3411-3443.

[5] T. Uchida, A. Tanigake, Y. Miyanaga, K. Matsuyama, M. Kunitomo, Y. Kobayashi, H. Ikezaki, A. Taniguchi, Evaluation of the bitterness of antibiotics using a taste sensor, J. Pharm. Pharmacol. 55 (2003) 1479-1485.

[6] J.Y.Zheng, M.P. Keeney, Taste masking analysis in pharmaceutical formulation development using an electronic tongue, Int. J. Pharm. 310 (2006) 118-124.

[7] A. Legin, A. Rudnitskaya, D. Clapham, B. Seleznev, K. Lord, Y. Vlasov, Electronic tongue for pharmaceutical analytics: quantification of tastes and masking effects, Anal. Bioanal. Chem. 380 (2004) 36-45

[8] A. Rudnitskaya, D. Kirsanov, Y. Blinova, E. Legin, B. Seleznev, D. Clapham, R.S. Ives, K.A. Saunders, A. Legin, Assessment of bitter taste of pharmaceuticals with multisensor system employing 3 way PLS regression, Anal. Chim. Acta 770 (2013) 45-52.

[9] D.H. Choi, N.A. Kim, T.S. Nam, S. Lee, S.H. Jeong, Evaluation of taste-masking effects of pharmaceutical sweeteners with an electronic tongue system, Drug Dev. Ind. Pharm. 40 (2014) 308-317

[10] K. Woertz, C. Tissen, P. Kleinebudde, J. Breitkreutz, Taste sensing systems (electronic tongues) for pharmaceutical applications, Int. J. Pharm. 417 (2011) 256-271.

[11] A. Gutés, D. Calvo, F. Céspedes, M. Del Valle, Automatic sequential injection analysis electronic tongue with integrated reference electrode for the determination of ascorbic acid, uric acid and paracetamol, Microchim. Acta 157 (2007) 1-6.

[12] E. Tokuyama, C. Matsunaga, K. Yoshida, J.C. Mifsud, T. Irie, M. Yoshida, T. Uchida, Famotidine orally disintegrating tablets: bitterness comparison of original and generic products, Chem. Pharm. Bull. 57 (2009) 382-387.

[13] K. Woertz, C. Tissen, P. Kleinebudde, J. Breitkreutz, Development of a taste-masked generic ibuprofen suspension: top-down approach guided by electronic tongue measurements, J. Pharm. Sci. 100 (2011) 4460-4470.
[14] A. Tanigake, Y. Miyanaga, T. Nakamura, E. Tsuji, K. Matsuyama, M. Kunitomo, T. Uchida, The bitterness intensity of clarithromycin evaluated by a taste sensor, Chem. Pharm. Bull. 51 (2003) 1241-1245.

[15] E. Tsuji, T. Uchida, A. Fukui, R. Fujii, H. Sunada, Evaluation of bitterness suppression of macrolide dry syrups by jellies, Chem. Pharm. Bull. 54 (2006) 310-314.

[16] M. Jańczyk, A. Kutyła, K. Sollohub, H. Wosicka, K. Cal, P. Ciosek, Electronic tongue for the detection of taste-masking microencapsulation of active pharmaceutical substances, Bioelectrochemistry 80 (2010) 94-98.

[17] M. Preis, M. Pein, J. Breitkreutz, Development of a taste-masked orodispersible film containing dimenhydrinate, Pharmaceutics 4 (2012) 551-562.

[18] N. Sadrieh, J. Brower, L. Yu, W. Doub, A. Straughn, S. MacHado, F. Pelsor, E. Saint Martin, T. Moore, J. Reepmeyer, D. Toler, A. Nguyenpho, R. Roberts, D.J. Schuirmann, M. Nasr, L. Buhse, Stability, dose uniformity, and palatability of three counterterrorism drugs - human subject and electronic tongue studies, Pharm. Res. 22 (2005) 1747-1756.

[19] C. Eckert, M. Pein, J. Reimann, J. Breitkreutz, Taste evaluation of multicomponent mixtures using a human taste panel, electronic taste sensing systems and HPLC, Sens. Actuator B-Chem. 182 (2013) 294-299.

[20] Y. Vlasov, A. Legin, A. Rudnitskaya, C. Di Natale, A. D’Amico, Nonspecific sensor arrays (electronic tongue) for chemical analysis of liquids: (IUPAC technical report), Pure Appl. Chem. 77 (2005) 1965-1983.

[21] M. del Valle, Electronic tongues employing electrochemical sensors, Electroanalysis 22 (2010) 1539-1555.

[22] K. Woertz, C. Tissen, P. Kleinebudde, J. Breitkreutz, A comparative study on two electronic tongues for pharmaceutical formulation development, J. Pharm. Biomed. Anal. 55 (2011) 272-281.

[23] M. Maniruzzaman, D. Douroumis, An in-vitro-in-vivo taste assessment of bitter drug: comparative electronic tongues study, J. Pharm. Pharmacol. 67 (1) (2015) 43-55.

[24] M. Pein, X.D. Gondongwe, M. Habara, G. Winzenburg, Interlaboratory testing of insent e-tongues, Int. J. Pharm. 469 (2014) 228-237.

[25] K. Woertz, C. Tissen, P. Kleinebudde, J. Breitkreutz, Performance qualification of an electronic tongue based on ICH guideline Q2, J. Pharm. Biomed. Anal. 51 (2010) 497-506

[26] M. Pein, C. Eckert, M. Preis, J. Breitkreutz, New protocol for $\alpha$ Astree electronic tongue enabling full performance qualification according to ICH Q2, J. Pharm. Biomed. Anal. 83 (2013) 157-163.

[27] J. Markiewicz, J. Taff, B. Wysocki, W. Wróblewski, Modular flow-through cell of the ion-selective electrode for water quality monitoring, date of application, 2008, P384364.

[28] E. Witkowska, A. Buczkowska, A. Zamojska, K.W. Szewczyk, P. Ciosek, Monitoring of periodic anaerobic digestion with flow-through array of miniaturized ion-selective electrodes, Bioelectrochemistry 80 (2010) 87-93.

[29] A. Kutyła-Olesiuk, M. Nowacka, M. Wesoły, P. Ciosek, Evaluation of organoleptic and texture properties of dried apples by hybrid electronic tongue, Sens. Actuators B Chem. 187 (2013) 234-240.

[30] A. Kutyła-Olesiuk, M. Zaborowski, P. Prokaryn, P. Ciosek, Monitoring of beer fermentation based on hybrid electronic tongue, Bioelectrochemistry 87 (2012) 104-113.

[31] P. Ciosek, M. Wesoły, M. Zabadaj, L. Lisiecka, K. Sołłohub, K. Cal, W. Wróblewski, Towards flow-through/flow injection electronic tongue for the analysis of pharmaceuticals, Sens. Actuators B: Chem. 207(PB (2015) 1087-1094.

[32] K.H. Esbensen, Multivariate data analysis - in practice, in: An Introduction to Multivariate Data Analysis and Experimental Design, 5th ed., CAMO ASA (norwegian software company), Oslo, 2001.

[33] A.K. Smilde, H.A.L. Kiers, S. Bijlsma, C.M. Rubingh, M.J. van Erk, Matrix correlations for high-dimensional data: the modified RV-coefficient, Bioinformatics 25 (2009) 401-405.

[34] U. Lorenzo-Seva, J.M.F. ten Berge, Tucker's congruence coefficient as a meaningful index of factor similarity, Methodology 2 (2006) 57-64.

[35] M. Preis, O. Haüsler, J. Breitkreutz, Electronic tongues in preformulation studies: application of electronic tongues in preformulation studies to evaluate taste-masking capacities of maltodextrins, Pharm. Ind. 76 (2014) 957-962.

[36] G.E. DuBois, Saccharin and cyclamate, in: Sweeteners and Sugar Alternatives in Food Technology, Wiley-Blackwell, 2012, pp. 137-166.

[37] B. Rezaei, M. Khalili Boroujeni, A.A. Ensafi, Caffeine electrochemical sensor using imprinted film as recognition element based on polypyrrole, sol-gel, and gold nanoparticles hybrid nanocomposite modified pencil graphite electrode, Biosens. Bioelectron. 60 (2014) 77-83.

[38] W.Y.H. Khoo, M. Pumera, A. Bonanni, Graphene platforms for the detection of caffeine in real samples, Anal. Chim. Acta 804 (2013) 92-97. 